\title{
A ND:YAG PUMPED BINARY LASER DYE MIXTURE FOR THE 397-427 NM WAVELENGTH REGION
}

\author{
J. L. FRENCH, J. KAROLCZAK ${ }^{a}$ and DENNIS J. CLOUTHIER ${ }^{b}$ \\ Department of Chemistry, University of Kentucky, Lexington, KY 40506-0055
}

(Received 27 January, 1994)

A useful mixture of the laser dyes DPS and bis-MSB in a 9:1 dioxane and methanol solution is described. The binary dye system exhibits broad tunability, symmetric degradation and reasonable conversion efficiency with $355 \mathrm{~nm}$ Nd:YAG pumping.

KEYWORDS: Laser dye mixture, dye laser, ultraviolet

\section{INTRODUCTION}

With the introduction of $355 \mathrm{~nm}$ ND:YAG and $308 \mathrm{~nm}$ excimer laser pumping, it is feasible to produce tunable pulsed dye laser radiation in the $330-420 \mathrm{~nm}$ nearultraviolet region of the spectrum. However, many of the dyes in this region exhibit limited tunability, short lifetimes and poor efficiency. The narrow tunability of many ultraviolet dyes makes spectroscopic investigations cumbersome and slow. Some recent publications (1-3) have demonstrated superior performance from $p$-terphenyl, $p$-quaterphenyl and similar dyes with $308 \mathrm{~nm}$ pumping. Landis et al. (4) have also reported two useful excimer pumped dye mixtures for the $340-380 \mathrm{~nm}$ region. In this communication, we briefly describe another inexpensive binary dye mixture which we have found useful in the $397-427 \mathrm{~nm}$ region with $355 \mathrm{~nm}$ ND:YAG pumping.

\section{EXPERIMENTAL DETAILS}

The dye mixture tests were conducted with a Lumonics HY 750 ND:YAG laser, using the $355 \mathrm{~nm}$ third harmonic to side pump a Lumonics HD 500 dye laser. The

a) Permanent Address: Quantum Electronics Laboratory, Physics Department, A. Michiewicz University, Grunwaldzka 6, 60-780 Poznan, Poland.

b) Correspondence Address: University of Kentucky, Department of Chemistry, Chemistry-Physics Building, Lexington, Kentucky 40506-0055. 


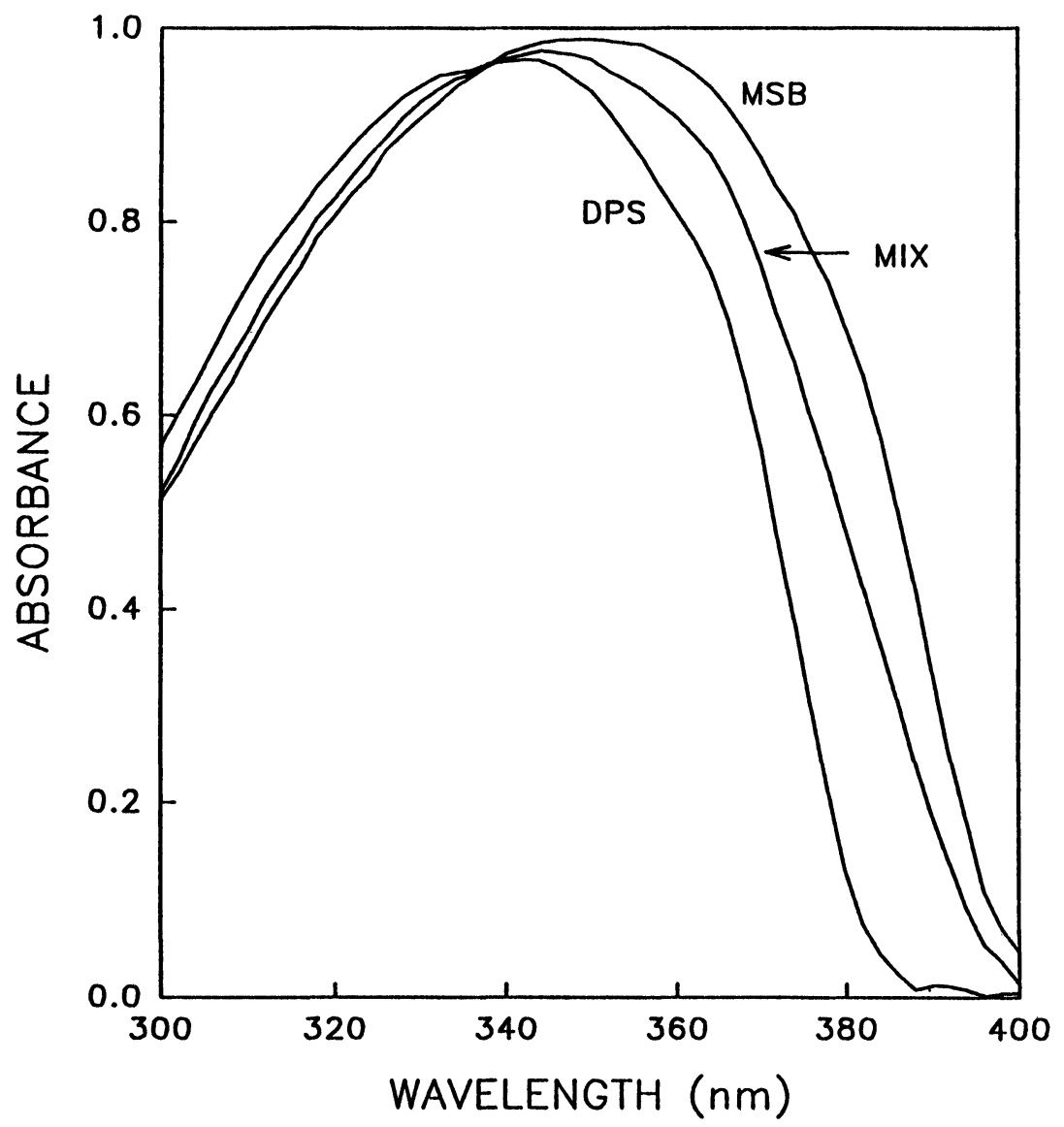

Figure 1 Absorption spectra of bis-MSB, DPS and the DPS/bis-MSB mixture. The concentrations of the dyes are $1.67 \times 10^{-5}$ (bis-MSB), $1.67 \times 10^{-5}$ (DPS) and $7.13 \times 10^{-6} / 8.94 \times 10^{-6} \mathrm{M}$ (DPS/bis-MSB)

dyes were circulated at the same concentration through the oscillator and amplifier of the dye laser. The laser dyes, DPS and bis-MSB, were obtained from Exciton chemical company and used as received. The solvent was a 9:1 mixture of $p$-dioxane (Aldrich) and HPLC grade methanol (Aldrich). Absorption spectra were taken on a Cary $1 \mathrm{UV}$-visible spectrophotometer using matched $1 \mathrm{~cm}$ pathlength quartz cuvettes.

The bis-MSB and DPS laser dyes absorb strongly in the 400-300 $\mathrm{nm}$ region with very similar absorption profiles, as illustrated in Fig. 1. DPS is shifted about $20 \mathrm{~nm}$ to shorter wavelengths relative to bis-MSB, suggesting that the two dyes might be useful as a binary mixture. In tests of the dye mixture to determine the optimum concentrations, we followed the procedure developed by Landis et al. (4) in their work on excimer-pumped dye mixtures. Initially, experiments were conducted to determine the appropriate molar ratios of the two dyes. A $1 \times 10^{-3} \mathrm{M}$ solution of DPS in the $p$-dioxane/methanol mixed solvent was tested for power and tunability. 
Then, $10 \mathrm{ml}$ portions of a saturated solution of bis-MSB were added to the DPS solution and the tuning curve and power recorded after each addition. The procedure was repeated, starting with $1 \times 10^{-4} \mathrm{M}$ bis-MSB and adding concentrated DPS. The optimum ratio was taken as that which gave the broadest tuning range with the most consistent power over the entire range. Subsequently, the molar concentration of the mixture was optimized. A very dilute solution of the mixture was prepared and the power spectrum was recorded. A concentrated solution of the mixture was then added in small aliquots until the power was optimized.

\section{RESULTS AND DISCUSSION}

The optimum concentration for this laser dye mixture was found to be $7.8 \times 10^{-5} \mathrm{M}$ in DPS and $9.8 \times 10^{-5} \mathrm{M}$ in bis-MSB, less than the optimum concentrations of the individual dye solutions. The absorption spectrum of the mixture is a smooth curve intermediate in range between those of the individual dyes, as shown in Fig. 1. The characteristics of the dye solutions and the binary mixture are reported in Table 1 and the individual tuning curves are shown in Fig. 2. The mixture has a full tuning range of $30 \mathrm{~nm}$ from $397-427 \mathrm{~nm}$ and has good power at $415 \mathrm{~nm}$, where neither of the individual dyes exhibit significant laser output. The lifetime (time to degrade to half-power with continual $355 \mathrm{~nm}$ pumping) of the mixture was measured to be 40.5 hours at $0.5 \mathrm{~W}$ or $72.9 \mathrm{~kJ} / \ell$, comparable to that of the single dye solutions. Most importantly, the output power of the mixture degrades symmetrically, so it is useful throughout its lifetime over the entire tuning range. The conversion efficiency for a freshly made solution was measured to be $12 \%$.

Table 1 Characteristics of laser dyes and the dye mixture

\begin{tabular}{lllll}
\hline Dye & $\begin{array}{l}\text { Optimum } \\
\text { concentration } \\
(\text { mol/e) }\end{array}$ & $\begin{array}{l}\text { Lasing } \\
\text { maximum } \\
(\mathrm{nm})\end{array}$ & \multicolumn{2}{c}{ Tuning range $(\mathrm{nm})$} \\
\cline { 4 - 5 } & $1.0 \times 10^{-4}$ & 406 & $401-411$ & $397-415$ \\
\hline DPS & $1.0 \times 10^{-4}$ & 421 & $416-425$ & $414-426$ \\
bis-MSB & $7.8 \times 10^{-5} / 9.8 \times 10^{-5}$ & $406 / 417$ & $400-426$ & $397-427$ \\
\hline DPS/bis-MSB & &
\end{tabular}

The result of this work is the formulation of a useful, time-saving binary dye mixture. Since the bis-MSB and DPS dyes are quite inexpensive, we have found this mixture to be of continuing utility despite the availability of newer, more efficient near-UV dyes (5). Its broad tuning range and symmetric degradation make it useful in a variety of situations requiring tunable $355 \mathrm{~nm}$ pumped ultraviolet laser radiation.

\section{Acknowledgements}

This research was supported by the National Science Foundation. 


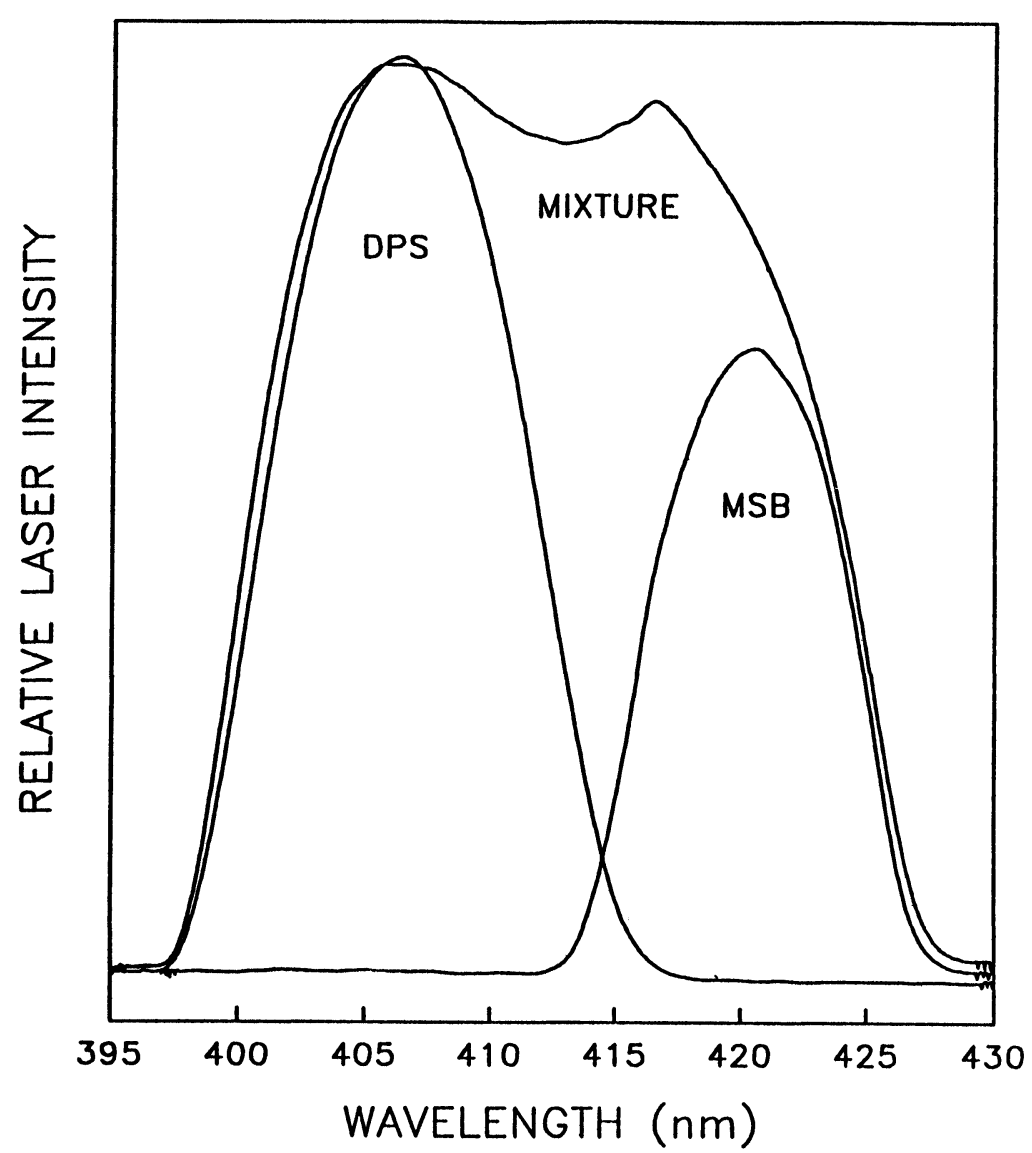

Figure 2 Tuning curves for the individual dyes and the DPS/bis-MSB mixture at the concentrations given in Table 1.

\section{References}

1. D. J. Schneider, D. A. Landis, P. A. Fleitz, C. J. Seliskar, J. M. Kauffman and R. N. Steppel, Laser Chem. 11, 49-62 (1991).

2. P. A. Fleitz, C. J. Seliskar, R. N. Steppel, J. M. Kauffman, C. J. Kelly and A. Ghiorghis, Laser Chem. 11, 99-107 (1991).

3. C. J. Seliskar, D. A. Landis, J. M. Kauffman, M. A. Aziz, R. N. Steppel, C. J. Kelly, Y. Qin and A. Ghiorghis, Laser Chem. 13, 19-28 (1993).

4. D. A. Landis, D. J. Schneider and C. J. Seliskar, Laser Chem. 11, 63-69 (1991).

5. Exciton Laser Dye Catalog, 1993. 